\title{
Entrainment of Slow Oscillations of Auditory Thalamic Neurons by Repetitive Sound Stimuli
}

\author{
Lixia Gao, ${ }^{1}$ Xiankai Meng, ${ }^{2}$ Changquan Ye, ${ }^{1}$ Haitian Zhang, ${ }^{1}$ Chunhua Liu, ${ }^{1}$ Yang Dan,,${ }^{3,4}$ Mu-ming Poo,,${ }^{1,3}$ Jufang He, ${ }^{2}$ \\ and Xiaohui Zhang ${ }^{1}$ \\ ${ }^{1}$ Institute of Neuroscience, State Key Laboratory of Neuroscience, Shanghai Institutes for Biological Sciences, Chinese Academy of Sciences, Shanghai \\ 200031, China, ${ }^{2}$ Department of Rehabilitation Sciences, Hong Kong Polytechnic University, Hong Kong, China, and ${ }^{3}$ Division of Neurobiology, Department \\ of Molecular and Cell Biology, Helen Wills Neuroscience Institute and ${ }^{4}$ Howard Hughes Medical Institute, University of California, Berkeley, Berkeley, \\ California 94720
}

Slow oscillations at frequencies $<1 \mathrm{~Hz}$ manifest in many brain regions as discrete transitions between a depolarized up state and a hyperpolarized down state of the neuronal membrane potential. Although up and down states are known to differentially affect sensoryevoked responses, whether and how they are modulated by sensory stimuli are not well understood. In the present study, intracellular recording in anesthetized guinea pigs showed that membrane potentials of nonlemniscal auditory thalamic neurons exhibited spontaneous up/down transitions at random intervals in the range of 2-30 s, which could be entrained to a regular interval by repetitive sound stimuli. After termination of the entraining stimulation (ES), regular up/down transitions persisted for several cycles at the ES interval. Furthermore, the efficacy of weak sound stimuli in triggering the up-to-down transition was potentiated specifically at the ES interval for at least $10 \mathrm{~min}$. Extracellular recordings in the auditory thalamus of unanesthetized guinea pigs also showed entrainment of slow oscillations by rhythmic sound stimuli during slow wave sleep. These results demonstrate a novel form of network plasticity, which could help to retain the information of stimulus interval on the order of seconds.

\section{Introduction}

Slow oscillations of neuronal membrane potential between the up and down states at frequencies $<1 \mathrm{~Hz}$ have been observed in various regions of the brain during slow wave sleep (Steriade et al., 1993a; Timofeev et al., 2001) or under anesthesia (Steriade et al., 1993b,c; Wilson and Kawaguchi, 1996; Anderson et al., 2000; Petersen et al., 2003; Sachdev et al., 2004). Even in awake animals, similar membrane potential fluctuations have been observed (Timofeev et al., 2001; Kitano et al., 2002; Petersen et al., 2003), but the durations of the up and down states tend to be shorter, typically on the order of tens of milliseconds (Steriade et al., 1993a; Timofeev et al., 2001; Destexhe and Sejnowski, 2003).

Slow oscillations in many brain regions interact strongly with sensory-evoked responses (Arieli et al., 1996; Petersen et al., 2003; Sachdev et al., 2004). For example, recordings from anesthetized or awake rats showed that whisker stimulation evoked larger depolarizing postsynaptic potentials and more spikes in cortical cells in the down state than in the up state (Petersen et al., 2003; Sachdev et al., 2004). In anesthetized cat V1, however, visual stimuli evoked larger depolarizations and more spikes in the neu-

Received Dec. 2, 2008; revised March 8, 2009; accepted April 2, 2009.

This work was supported by grants from the Knowledge Innovation Program of the Chinese Academy of Sciences (KSCX2-YW-R-29). We thank Feng Yu for help with some data analysis.

Correspondence should be addressed to either of the following: Jufang He, Department of Rehabilitation Sciences, Hong Kong Polytechnic University, Hung Hom, Kowloon, Hong Kong, China, E-mail: Jufang.He@inet. polyu.edu.hk; or Xiaohui Zhang, Institute of Neuroscience, Chinese Academy of Sciences, 320 Yue-Yang Road, Shanghai 200031, China, E-mail: xhzhang@ion.ac.cn.

DOI:10.1523/JNEUROSCI.5733-08.2009

Copyright $\odot 2009$ Society for Neuroscience $\quad$ 0270-6474/09/296013-09\$15.00/0 rons that were already depolarized (Azouz and Gray, 1999). Thus, slow membrane potential oscillations can significantly affect sensory information processing, in a manner that varies among different sensory circuits. Conversely, sensory stimulation can also affect the temporal pattern of slow oscillations. For example, visual stimulation increased the duration of the up state in cat cortical neurons (Anderson et al., 2000), and air-puff stimulation of the rat vibrissae evoked either up-to-down or down-to-up membrane potential transition in cerebellar Purkinje cells (Loewenstein et al., 2005; Schonewille et al., 2006). In the auditory thalamus, slow oscillations of neuronal activity have been observed in anesthetized animals, and extracellular recordings showed that repetitive sound stimulation could elicit regular oscillations of thalamic neurons ( $\mathrm{He}, 2003)$. Although the interactions between sensory stimulation and ongoing network oscillations are likely to play important roles in various brain functions, experimental characterization of these interactions remains incomplete.

In the present study, we performed in vivo recordings in both anesthetized and unanesthetized animals to examine the effect of rhythmic sound stimuli on slow oscillations of auditory thalamic neurons. Intracellular recordings from nonlemniscal thalamic neurons in anesthetized guinea pigs showed that the transition of membrane potentials from up to down state could rapidly timelock to the rhythmic sound stimuli with interstimulus intervals (ISIs) on the order of seconds. Extracellular recordings showed that such entrainment of slow oscillations also occurred in the auditory thalamus of unanesthetized guinea pigs during slow wave sleep. Furthermore, we found that after termination of the 
sound stimulation, the regular up/down transitions at the entrained interval persisted for tens of seconds, and the efficacy of sound stimuli in evoking up to down membrane potential transitions was enhanced specifically at the entrained interval for at least $10 \mathrm{~min}$. These findings suggest that slow oscillations in the auditory thalamus may help to retain the information of rhythmic stimulus intervals on the order of seconds.

\section{Materials and Methods}

Animal preparation. Adult guinea pigs (8-10 weeks, 350-700 g) were used in this study. For intracellular recordings, animals were initially anesthetized with pentobarbital sodium (Nembutal, $40 \mathrm{mg} \mathrm{kg}^{-1}$, i.p.; Abbott Laboratories) and maintained by the same anesthetic (5-10 mg $\mathrm{kg}^{-1} \mathrm{~h}^{-1}$ ) during the surgical procedure and recording. The animal was then paralyzed with Gallamine triethiodide (initially $50 \mathrm{mg} \mathrm{kg}^{-1}$, maintained at $5-10 \mathrm{mg} \mathrm{kg}^{-1} \mathrm{~h}^{-1}$, i.m.) and artificially ventilated with a respirator. After mounted in a stereotaxic device, a craniotomy was performed to enable access to the medial geniculate body (MGB) in the right hemisphere, and CSF was released at the medulla level. Throughout the intracellular recording experiment, the core body temperature $\left(37-38^{\circ} \mathrm{C}\right)$, heart rate, and electroencephalogram (EEG) were continuously monitored to assess the level of anesthesia.

For extracellular recordings, adult guinea pigs were anesthetized with a mixture of ketamine $(40 \mathrm{mg} / \mathrm{kg}$, s.c. $)$ and xylazine $(10 \mathrm{mg} / \mathrm{kg}$, s.c. $)$ for the surgical implantation of tetrodes on a stereotaxic apparatus (Narishige). A small craniotomy was made on top of the MGB in the right hemisphere [Bregma coordinate $(-5.6,+4.0)$ ], followed by removal of the dura. The tetrode was slowly lowered with a microdrive (Narishige) into the MGB, and neural activity was monitored to help ensure proper placement of the tetrode. Sterile agar ( $4 \%$ in saline) and dental cement were applied onto the craniotomy and the electrode socket to fix the electrode with two screws on the left parietal skull. Two other stainless screws were implanted in the left frontal and left parietal skull to record EEG. An electromyogram (EMG) wire was implanted in the dorsal neck muscles. After the surgery, the guinea pig was transferred to a recovery cage, and buprenorphine $(0.15 \mathrm{mg} / \mathrm{kg}$ s.c. every $8 \mathrm{~h})$ was administered, if necessary. The animal use protocol was approved by the Animal Use Committee of Shanghai Institutes for Biological Sciences, Chinese Academy of Sciences.

Intracellular recordings in anesthetized animals. Intracellular recordings from auditory thalamic neurons were performed with sharp glass micropipettes. In the majority of experiments, the pipettes were filled with $3.0 \mathrm{M} \mathrm{K}^{+}$acetate (KAc, $3.0 \mathrm{M}, \mathrm{pH} 7.6$ ); in a subset of experiments for examining ionic mechanisms of membrane potential oscillations, cesium acetate $(\mathrm{CsAc}, 3.0 \mathrm{M})$ or $\mathrm{K}^{+}$chloride $(\mathrm{KCl}, 1.0 \mathrm{M})$ was used. The electrode (resistance, 40-90 M $\Omega$ ) was advanced vertically with a motorized manipulator (Narishige). After the electrode was lowered to a depth of 4-5 $\mathrm{mm}$, the cortical exposure was sealed using low-melting temperature paraffin. When the electrode was near or in the target area, it was slowly advanced at 1 or $2 \mu \mathrm{m}$ per step. The electrical signals were amplified by an Axon 2A amplifier (Molecular Devices), digitalized by DIGIDATA converter board (1322A; Molecular Devices), and then stored in the computer with the AxoScope software. Data from neurons with resting membrane potential below $-50 \mathrm{mV}$ and spontaneous spike amplitude $>50$ $\mathrm{mV}$ were included in the study.

Extracellular recordings in unanesthetized animals. A tetrode made from nichrome microwires (California Fine Wire) was implanted in the MGB of the right hemisphere for extracellular recording of neuronal activity in free-moving adult guinea pigs (see above). After 5-7 d of accommodation, extracellular recordings of MGB neurons were performed in a soundproof box, where the guinea pig could move freely. In some experiments, multiunit spiking activities and local field potentials (LFPs; with a $0.1-200 \mathrm{~Hz}$ band filter) were recorded simultaneously. The electrical signals (including EEG and EMG) were amplified by a TuckerDavis Technologies (TDT) Medusa multichannel amplifier (TuckerDavis Technologies), digitalized, and analyzed with the pClamp software (Molecular Devices).

Data analysis. To determine the mean membrane potentials of the up and down states for each thalamic cell, histograms of membrane potential distribution over a randomly selected $25 \mathrm{~s}$ recording period were constructed, and the two peaks in the distribution were used as the mean membrane potentials for the up and down states. The transitions between the up and down states were detected using a method described previously (Loewenstein et al., 2005). In brief, two thresholds were set at one-fourth and three-fourths of the distance between the mean membrane potentials. When the membrane potential rose above the lower threshold, a down-to-up transition was registered; when the membrane potential fell below the upper threshold, an up-to-down transition was registered. The duration of each state was defined as the time between each pair of consecutive transitions. The mean up- and down-state durations of each cell were computed by averaging over a continuous recording of 25-940 s.

Data are presented as mean \pm SEM, unless otherwise indicated, and statistical significance was tested with paired $t$ test in most cases. For data with abnormal distributions, statistical significance was tested with Wilcoxon signed-rank test instead.

Sound stimulation. Sound stimuli were generated digitally by a TDT system (Tucker-Davis Technologies) and delivered either to the left ear of the anesthetized animal through an ear bar containing a drilled hole or to the open field speakers in the box for the awake animal. The sound pressure level (SPL) was calibrated with a condenser microphone (Center Technology) over the frequency range of $100 \mathrm{~Hz}$ to $35 \mathrm{kHz}$. Noise bursts (over the entire frequency range, duration $200 \mathrm{~ms}$ ) were used as experimental stimuli. The experiments were performed in a soundproofed room or box.

Anatomical studies. To confirm the location of the recorded neuron, the recording electrode was filled with 1-2\% neurobiotin (VectorLaboratories) in $3.0 \mathrm{M} \mathrm{KAc}$. Neurobiotin was injected into one or two neurons in each animal by passing depolarizing pulses $(2 \mathrm{nA}, 150 \mathrm{~ms} /$ pulse at 3.3 $\mathrm{Hz}$ for 1-4 min) after the recording. A total of nine animals were used for anatomical confirmation. After the tracer injection, the animal was deeply anesthetized with sodium pentobarbital $\left(40 \mathrm{mg} \mathrm{kg}^{-1}\right)$ and perfused transcardially with $0.9 \%$ saline followed by a mixture of $4 \%$ paraformaldehyde in a $0.1 \mathrm{~m}$ phosphate buffer, $\mathrm{pH}$ 7.3. The brains were removed and postfixed in $4 \%$ paraformaldehyde overnight, then transferred to a $0.1 \mathrm{M}$ phosphate buffer containing $30 \%$ sucrose. The thalamus and the brainstem were cut transversally using a freezing microtome (Leica) at a thickness of $50 \mu \mathrm{m}$. Serial sections of the thalamus were collected in $0.01 \mathrm{M}$ potassium PBS (KPBS), $\mathrm{pH} 7.4$, and incubated in $0.1 \%$ peroxidase-conjugated avidin-D (Vector Laboratories) in KPBS with $0.5 \%$ Triton X-100 for $4-6 \mathrm{~h}$ at room temperature. After detection of peroxidase activity with $3^{\prime}, 3^{\prime}$-diaminobenzidine (DAB), sections were examined under the microscope and photographed. Those sections containing labeled neurons were mounted on gelatin-coated slides and counterstained with Neutral Red (1\%; Sigma).

\section{Results}

\section{Bistable membrane potentials of auditory thalamic neurons in vivo}

Neuronal activity in the auditory thalamus of anesthetized adult guinea pigs was recorded intracellularly with sharp electrodes. Approximately two-thirds of the 174 recorded neurons exhibited spontaneous slow membrane potential oscillations between the up and down states (Fig. $1 B$ ), resulting in a bimodal membrane potential distribution (Fig. 1C) (Wilson and Kawaguchi, 1996; Timofeev et al., 2001; Petersen et al., 2003). Spiking occurred primarily at the up state (Fig. $1 B$ ). Neurobiotin labeling (Fig. $1 A$ ) indicated that 2 of 4 cells recorded in the ventral division (lemniscal nucleus) and 4 of 5 cells in the dorsal and caudomedial divisions (nonlemniscal nucleus) of the MGB showed up and down states, suggesting that slow oscillations are more prominent in the nonlemniscal nucleus. Similar to that reported previously (He, 2003), we found that the auditory response latency of the nonlemniscal neurons $(29.0 \pm 15.7 \mathrm{~ms}, \mathrm{SD}, n=5)$ was longer than that of the lemniscal cells $(15.2 \pm 3.8 \mathrm{~ms}, n=4)$ in the 


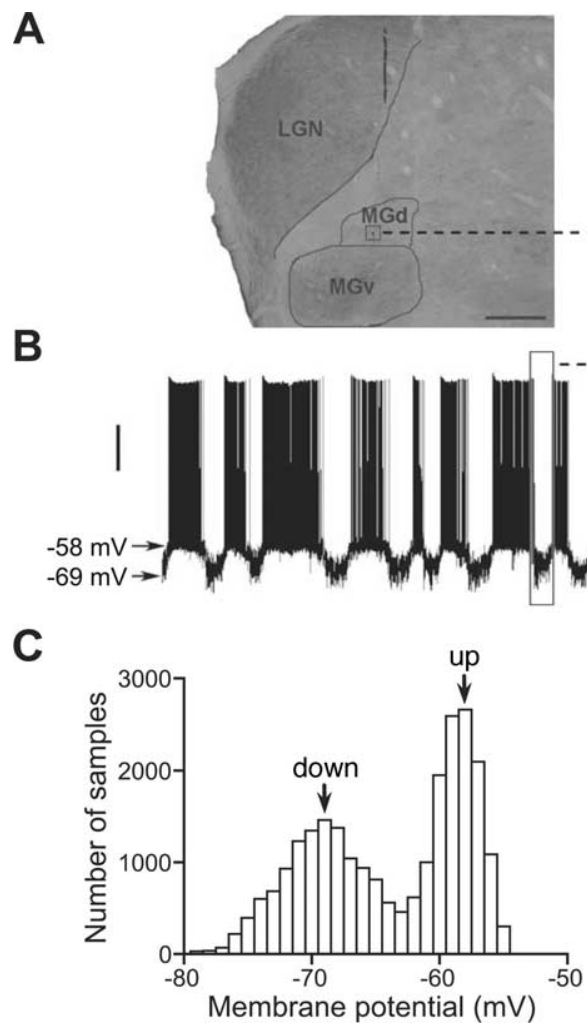

E

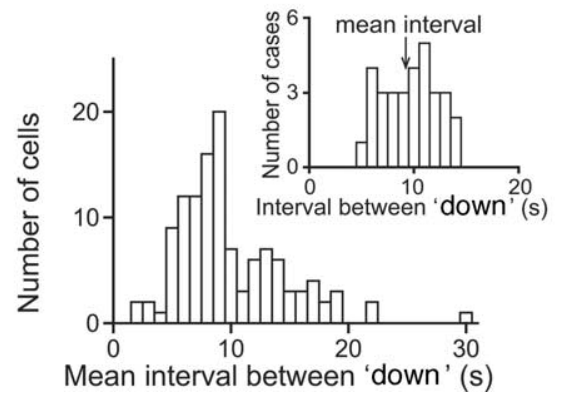

Figure 1. Spontaneous slow oscillation of auditory thalamic neurons between up and down states. $\boldsymbol{A}$, Image of a DAB-stained section of guinea pig thalamus containing a recorded neuron labeled with neurobiotin. Subdivisions of thalamus: MGv, ventral nucleus of MGB; MGd, dorsal nucleus of MGB; LGN, lateral geniculate nucleus. Boxed region is shown at a higher resolution. Scale bars: left, $500 \mu \mathrm{m}$; right, $20 \mu \mathrm{m}$. $\boldsymbol{B}$, Example recording of spontaneous up/down transitions in an MGB neuron. The segment in gray rectangle is expanded and shown on the right. Calibration: $20 \mathrm{mV}, 10 \mathrm{~s}$ (left), $1 \mathrm{~s}$ (right). C, Distribution of membrane potentials over a $25 \mathrm{~s}$ recording period for the neuron shown in $\boldsymbol{B}$, binned at $1 \mathrm{~ms}$. Arrows, mean membrane potentials in up $(-58$ $\mathrm{mV})$ and down $(-69 \mathrm{mV})$ states. For a population of cells, the average membrane potentials of up and down states were $-58.3 \pm 5.0 \mathrm{mV}(\mathrm{SD})$ and $-69.4 \pm 5.5 \mathrm{mV}$, respectively. D, Difference between the average membrane potentials in up and down states for 121 neurons. $\boldsymbol{E}$, Distribution of mean intervals between consecutive down states (arrow in inset) for the same 121 neurons. Inset, Distribution of intervals for the neuron shown in $\boldsymbol{B}$. Arrow, mean value. $\boldsymbol{F}$, Distribution of durations of up and down states for the same 121 neurons.

population of neurobiotin-labeled neurons. We thus used response latency as a criterion to select two groups of cells from the recorded pool: lemniscal cell-like (with latency $<10 \mathrm{~ms}$ ) and nonlemniscal cell-like (with latency $>20 \mathrm{~ms}$ ). We found that 3 of 25 lemniscal-like cells and 30 of 34 nonlemniscal-like cells exhibited slow oscillations. Thus, slow oscillation is indeed more prominent in nonlemniscal than lemniscal neurons.

For each cell exhibiting slow oscillations, we determined the mean membrane potentials at the up and down states by identifying two distinct peaks in the membrane potential distribution (Fig. $1 C$, arrows). The difference between them ranged from 4 to $20 \mathrm{mV}$ (mean $\pm \mathrm{SD}, 11.1 \pm 3.4 \mathrm{mV} ; n=121$ cells) (Fig. $1 D$ ), similar to that found in neocortical neurons (Steriade et al., 1993b; Timofeev et al., 2001; Petersen et al., 2003), cerebellar Purkinje cell (Loewenstein et al., 2005), and neostriatal spiny neurons (Wilson and Kawaguchi, 1996). The average interval between the onset times of two adjacent down states (equivalent to the sum of the mean durations of up and down states) was $10.1 \pm$ $5.7 \mathrm{~s}$ (SD, $n=121$; range, 2 to $30 \mathrm{~s}$ ) (Fig. $1 E)$, consistent with the frequency of previously observed slow oscillations in auditory thalamic neurons (He, 2003). For each given cell, the interval was highly variable (Fig. $1 E$, inset). Most of this variability resulted from the variable duration of the up state $(6.2 \pm 5.0 \mathrm{~s}, \mathrm{SD}, n=121)$, whereas the duration of the down state was more narrowly distributed $(3.9 \pm 2.2 \mathrm{~s})$ (Fig. 1F).

\section{Auditory stimuli evoke up-to-down transitions}

Previous studies showed that the responses of cortical neurons to sensory stimuli depend on whether the neuron is in the up or down state (Petersen et al., 2003), and the transition between the two states can be modulated by sensory inputs (Anderson et al., 2000; Loewenstein et al., 2005). We, therefore, tested the effect of auditory stimuli on the membrane potential of thalamic neurons. We found that the presentation of a brief sound stimulus (noise burst with a duration of $200 \mathrm{~ms}$ ) triggered an up-to-down transition when the neuron was in the up state (Fig. 2A) but had no effect in the down state (Fig. $2 B)$. Repetitive sound stimulation at a fixed ISI caused periodic downward transitions in phase with the onset of sound stimuli (Fig. 2A), a process referred to as "entrainment." The average durations of both up and down states increased with the ISI, although the duration of the up state was more strongly affected (Fig. 2C) $(n=14)$. When sound stimuli with various ISIs were presented, we found significant entrainment within the range of 3 to $12 \mathrm{~s}$ (Fig. $2 D)(n=14)$. The amplitude of the downward transition increased with the sound intensity, reaching saturation at $\sim 30 \mathrm{~dB}$ SPL (Fig. $2 E, F$, sound pressure level).

\section{Ionic basis of down-state hyperpolarization}

Slow oscillations in the thalamus involve activity of the recurrent network between the neocortex and the thalamus (Steriade et al., 1993a,c). In particular, thalamic reticular nucleus (TRN), a thin network that provides strong inhibitory inputs to thalamocortical projection neurons, is known to modulate thalamic oscillations (Huntsman et al., 1999; Bal et al., 2000; Steriade, 2001; Timofeev et al., 2001). To test the idea that GABAergic inhibitory synaptic inputs from TRN are responsible for the down state of MGB neurons, we examined the ionic basis of the thalamic 
down-state hyperpolarization. When the normal intrapipette KAc solution was replaced with $\mathrm{KCl}$ solution, which shifts the reversal potential of $\mathrm{Cl}^{-}$current to a more positive level, 13 of 18 (72\%) of the MGB neurons recorded exhibited a marked shift of the down-state membrane potential to more depolarized levels under both spontaneous (Fig. $3 A, C, G$ ) and soundstimulated (Fig. $3 B, D, H$ ) conditions, leading to reversal of the up/down polarity. Thus, the down state was strongly affected by the internal $\mathrm{Cl}^{-}$concentration. In contrast, the down state of these thalamic neurons was much less affected when the pipette was filled with a high concentration of $\mathrm{Cs}^{+}$(Fig. $3 E, F$ ), a nonspecific intracellular blocker of $\mathrm{K}^{+}$channels. This differs from the down state of cortical neurons, which depends strongly on $\mathrm{K}^{+}$channel activity (Timofeev et al., 2001). Figure 3, $G$ and $H$, summarizes the up-to-down membrane potential changes for all the cells recorded with pipettes filled with $\mathrm{KAc}, \mathrm{KCl}$, or CsAc. Compared with that for KAc, significant positive shifts were found for the $\mathrm{KCl}$ but not for the CsAc condition. Thus, the down state associated with slow oscillations of the nonlemniscal thalamic neurons is caused by inhibitory synaptic inputs, presumably from the TRN or local GABAergic neurons within the MGB.

\section{Entrainment of slow oscillations in unanesthetized animals}

To determine whether the entrainment of thalamic activity by rhythmic sound stimulation depends on anesthesia, we performed in vivo tetrode recordings from MGB of unanesthetized guinea pigs. During the recording sessions, the animals underwent natural sleep/awake circles, as indicated by EEG and EMG. As shown by the sample recordings in Figure $4 A_{1}$, slow wave sleep is characterized by EEG activity of higher amplitude and lower frequency and EMG activity of lower amplitude than those observed in the awake state.

Power spectral analysis of MGB spiking activity (Fig. $4 \mathrm{~A}_{2}$ ) and LFP (supplemental Fig. 1, available at www.jneurosci.org as supplemental material) showed that spontaneous activity during slow wave sleep was entrained by the entraining stimulation (ES) with an ISI of $3 \mathrm{~s}$, as indicated by the sharp peak at the ES frequency $(0.33 \mathrm{~Hz})$. Similar entrainment was induced by repetitive sound stimuli at ISIs within the range of $1-4 \mathrm{~s}$ (Fig. $4 A_{2}, B$, arrows). In the awake state, we did not observe entrainment of the MGB activity by the ES at an ISI of $3 \mathrm{~s}$, in either multiunit activity (Fig. $4 A_{2}$ ) or LFPs (supplemental Fig. 1, available at www.jneurosci.org as supplemental material). Together, these results indicate that the entrainment of neural activity in the auditory thalamus by rhythmic sound stimuli was not caused by anesthesia, al-
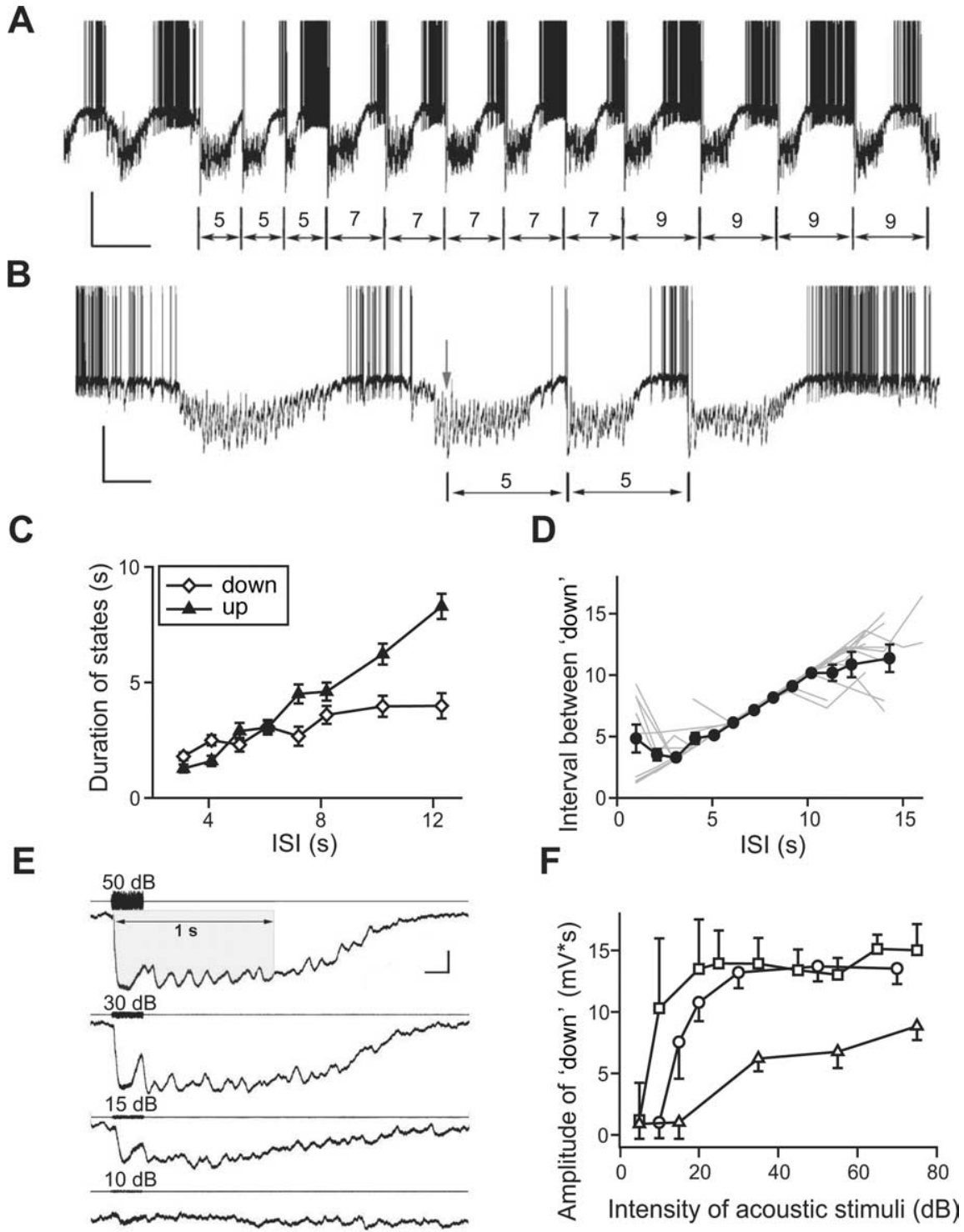

Figure 2. Up-to-down transitions induced by sound stimulation. $\boldsymbol{A}$, An example recording of membrane potentials in response to sound stimuli (noise bursts, intensity $75 \mathrm{dBSPL}$, duration $200 \mathrm{~ms}$, indicated by lines below) at intervals of 5, 7, and $9 \mathrm{~s}$. Note that each sound stimulus reliably induced an up-to-down transition. Calibration: $20 \mathrm{mV}, 7 \mathrm{~s}$. $\boldsymbol{B}$, Another example recording showing that sound stimuli did not cause down-to-up transitions when given in the down state. Gray arrow indicates the time of the first stimulus. Calibration: $20 \mathrm{mV}, 2$ s. C, Mean duration of each state plotted against the interval between sound stimuli. D, Mean interval between consecutive down states versus ISI of sound stimuli. Gray lines represent data from individual neurons. Black line represents average across all neurons. Error bars indicates SEM. $\boldsymbol{E}$, Membrane potential responses to 50, 30, 15, and $10 \mathrm{~dB}$ SPL noise bursts of an example cell. Each trace was averaged from seven trials. Calibration: $5 \mathrm{mV}, 150 \mathrm{~ms}$. F, Amplitude of response (measured by area over $1 \mathrm{~s}$, shaded area in $\boldsymbol{E}$ ) evoked by different sound intensities $(n=3)$. Each symbol represents one cell. Error bars indicate SD. The cell depicted by triangles appeared to be a fast-spiking interneuron based on its spiking characteristics, bars indicate SD. The cell depicted by triangles appeare
whereas the other two were regular-spiking neurons.

though the effect is more prominent during slow wave sleep than in the awake state.

\section{Effect of entrainment on spontaneous up/down transition}

We next examined the effect of entrainment by rhythmic sound stimuli on subsequent spontaneous up/down transitions of the nonlemniscal neurons in anesthetized animals. As shown by an example recording in Figure 5, $A$ and $B$, ES with an ISI of $8 \mathrm{~s}$ resulted in a gradual change of up-to-down transitions from highly variable intervals ( 4 to $14 \mathrm{~s}$ ) to a narrow range around the entrained ISI. After termination of the ES, the spontaneous transitions occurred with intervals close to the entrained ISI for tens 


\section{spontaneous up-down states}

A

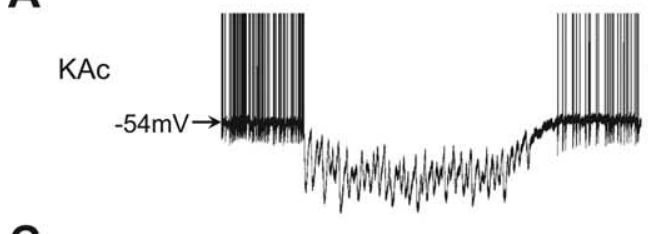

C

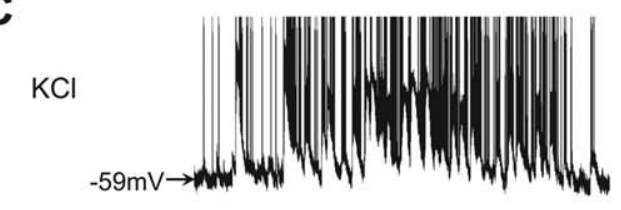

E

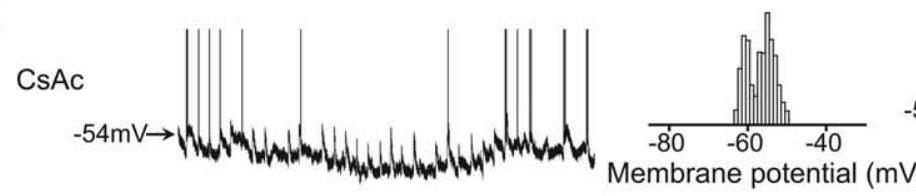

G
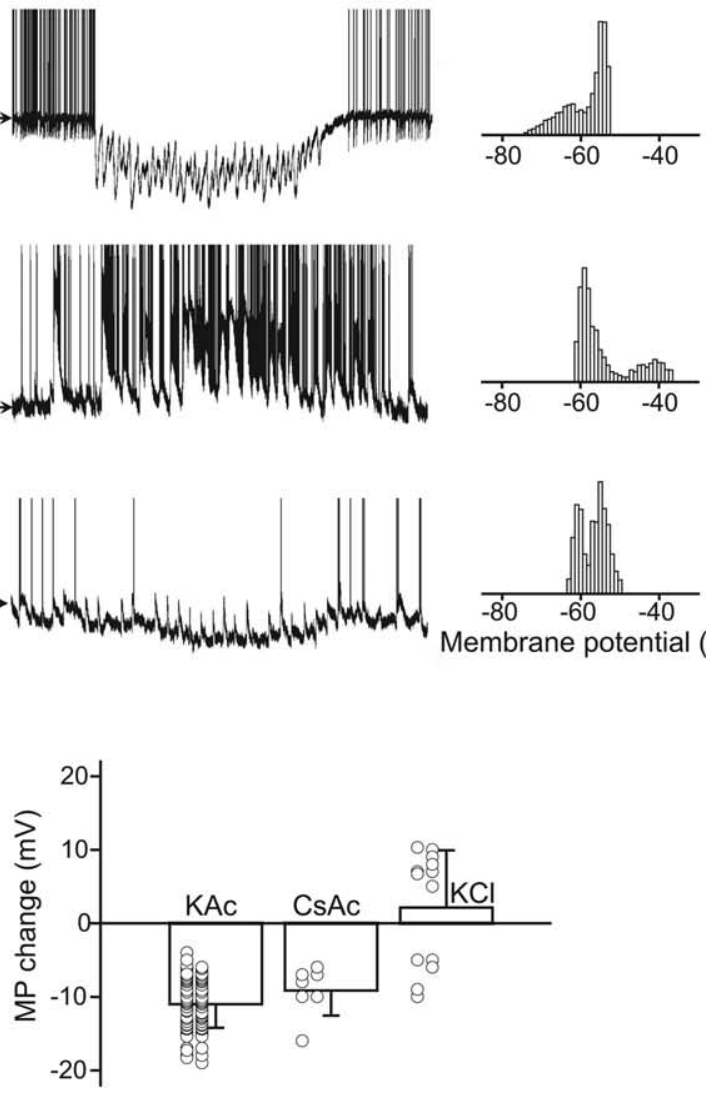
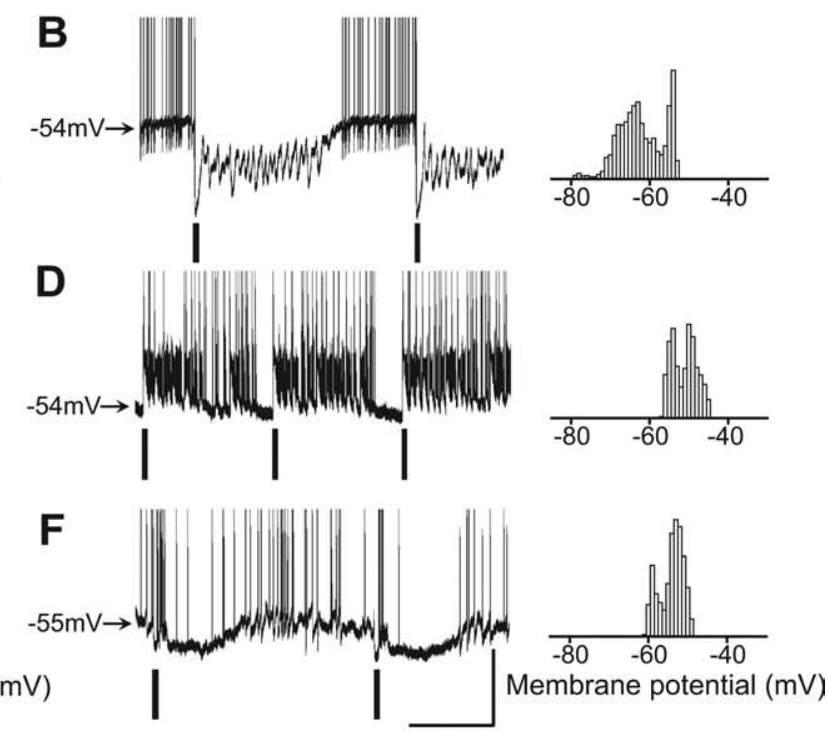

H

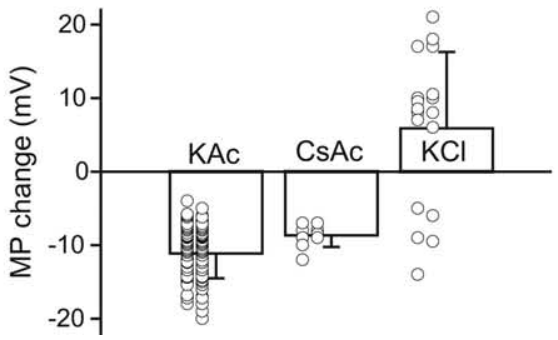

Figure 3. Ionic basis of down-state hyperpolarization. $\boldsymbol{A}-\boldsymbol{F}$, Intracellular recording of membrane potentials and histograms of membrane potential distributions in the spontaneous $(\boldsymbol{A}, \boldsymbol{C}, \boldsymbol{E})$ and sound stimulation $(\boldsymbol{B}, \boldsymbol{D}, \boldsymbol{F})$ periods from the neurons recorded by a micropipette filled with three different solutions. Calibration: $20 \mathrm{mV}, 4 \mathrm{~s}$. $\boldsymbol{G}, \boldsymbol{H}$, Summary of mean membrane potential changes in the spontaneous $(\boldsymbol{G})$ and sound stimulation $(\boldsymbol{H})$ periods for the neurons recorded using three different pipette solutions. Each circle represents data from one recorded neuron. Error bars indicate SD.

of seconds before it became highly variable again (Fig. $5 B$ ). When we divided the neurons into two groups (Fig. $5 C$ ), those with ISI of ES longer ( $n=7$, solid triangles) or shorter $(n=14$, open triangles) than the mean interval of spontaneous transitions before the ES, we found that the ES prolonged or reduced, respectively, the intervals of spontaneous transitions for several cycles after termination of the ES. This is also shown by the changes in the mean interval of individual neurons in these two groups (Fig. $5 D)$. Within seven cycles after the ES, the variability of the intervals, as measured by the coefficient of variation (CV; defined as $\mathrm{SD} / \mathrm{mean})$, was significantly reduced (Fig. $5 E)(n=21, p<0.05)$. Thus, the ISI of sound stimuli was retained in the spontaneous up/down transitions of these thalamic neurons for a period of tens of seconds after the entrainment. The retention of the ES interval in the spontaneous oscillatory activity after the cessation of rhythmic sound stimuli was also found in the thalamus of unanesthetized guinea pig brain (supplemental Fig. 2, available at www.jneurosci.org as supplemental material).

\section{Interval-specific potentiation of responses to weak stimuli}

In addition to the brief retention of the ES interval in the spontaneous up/down transitions, we also examined the effect of ES on the efficacy of subsequent sound stimuli in triggering the upto-down transitions. The efficacy of each weak test stimulus (20 $\mathrm{dB}$ weaker than the ES) was measured by the mean amplitude of the down response within $1 \mathrm{~s}$ after the stimulus (Fig. $2 E$ ). When the efficacy for test stimuli at various intervals (test-ISI, from a pool of $2,3,5,7$, or $9 \mathrm{~s}$ presented at a random sequence in each trial) was compared before and after five blocks of ES (five strong stimuli per block at ES-ISI of $5 \mathrm{~s}$ ) (Fig. 6A), we found that the efficacy was increased at the test interval of $5 \mathrm{~s}$ but not at 2,3,7, or $9 \mathrm{~s}$ (Fig. $6 B_{2}$ ). Interestingly, when the efficacy was plotted as a function of residue time (shortest interval between the test stimulus and an anticipated ES) (Fig. $6 B_{1}$ ) rather than the test-ISI, we found no temporal specificity in the potentiation (Fig. $6 B_{3}$ ). This indicates that for the timing-specific potentiation, time was not counted by a fixed clock set by the ES. Instead, each test stimulus resets the timing mechanism. Such interval-specific potentiation was also observed for ES with an ISI of 8 or $10 \mathrm{~s}$ (supplemental Fig. 3, available at www.jneurosci.org as supplemental material). Finally, we examined the persistence of the interval-specific potentiation by measuring the efficacy at $0,5,10,15$, and $20 \mathrm{~min}$ after ES termination (Fig. $6 \mathrm{~A}$, decay time test). We found that the effect decayed slowly over a period of $20 \mathrm{~min}$ (Fig. 6C,D), suggesting that slow oscillation in these auditory thalamic neurons is capable of retaining the information of stimulus interval for a period of at least $10 \mathrm{~min}$.

\section{Discussion}

In the present study, using intracellular recording from anesthetized guinea pigs, we found that rhythmic sound stimulation at an interval of a few seconds can entrain up-to-down transitions of 
A1

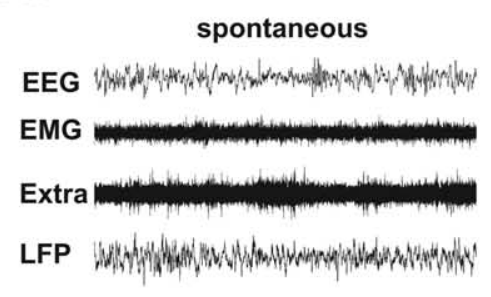

$\mathrm{A}_{2}$

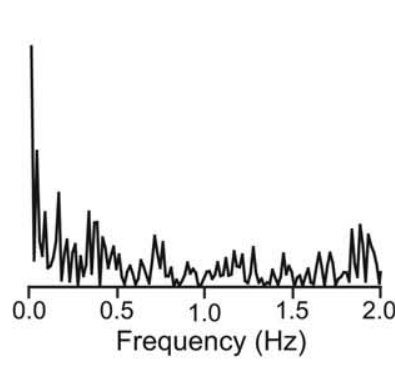

Slow wave sleep

evoked

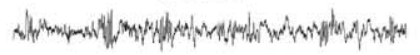

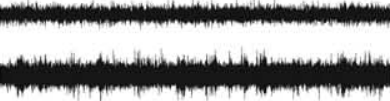

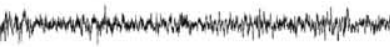

Awake state

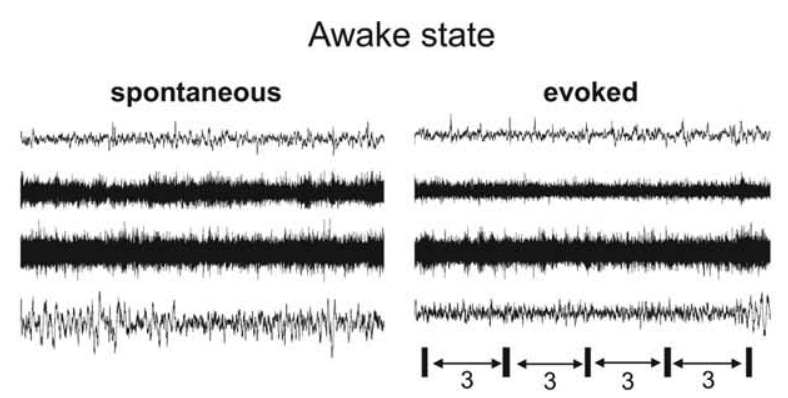

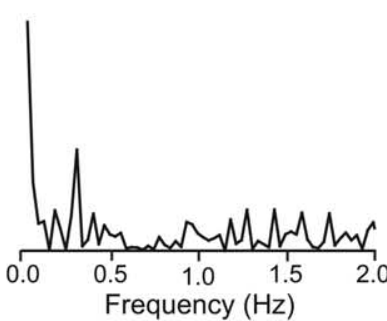

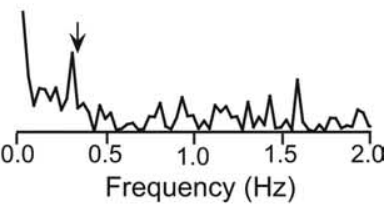

B

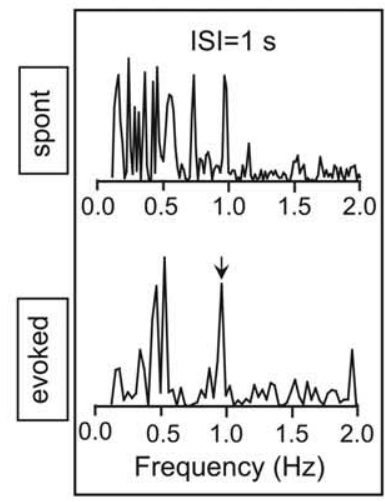

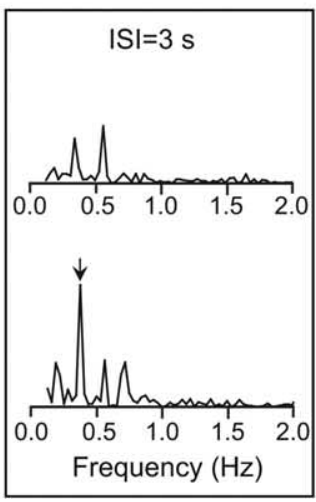

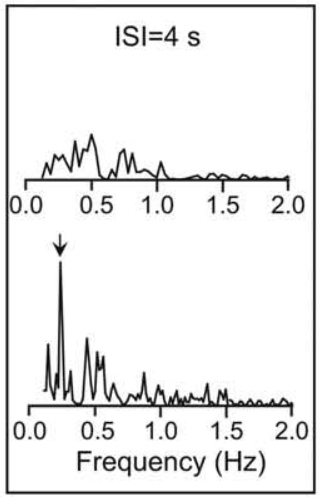

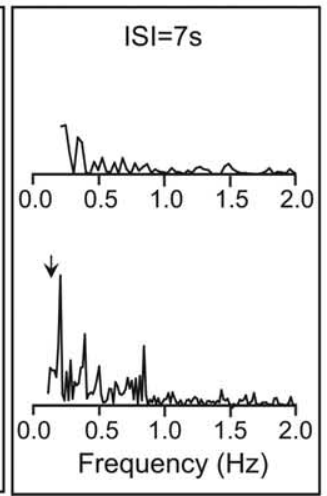

Figure 4. Entrainment of slow oscillations in unanesthetized guinea pigs. $A$, An example extracellular recording and power spectrum of auditory thalamic neurons during sleep/awake circles. $A_{1}$, Sample EEG and EMG recordings, spiking activity, and LFPs in the auditory thalamic neurons recorded by implanted tetrode before (spontaneous) and during (evoked) repetitive sound stimulation $(I S I=2 s)$, during slow wave sleep and in the awake state. Calibration: $2 \mathrm{~s} . \boldsymbol{A}_{2}$, Power spectrum analysis of spiking activity shown in $\boldsymbol{A}_{1}$. The spectra were calculated from the spike rates with an fast Fourier transform algorithm, and the arrows mark the expected frequencies of the entrained oscillation. $B$, Power spectrum analysis of the spiking activity recorded during slow wave sleep in five different experiments before (spont) and during (evoked) repetitive sound stimuli at different ISIs $(1,3,4,5$, and $7 \mathrm{~s})$. The results are presented in the same manner as in $\boldsymbol{A}_{2}$.

the membrane potential of nonlemniscal auditory thalamic neurons. Moreover, post-ES spontaneous transitions occurred at the entrained interval for a few cycles, suggesting a short-term memory in the neural network. Further studies revealed a longer-lasting effect, in which the efficacy of weak sound stimuli in evoking up-todown transitions was potentiated specifically at the entrained interval for at least $10 \mathrm{~min}$. Importantly, extracellular recording in unanesthetized animals showed a similar entrainment of slow oscillations by rhythmic sound stimulation during slow wave sleep, indicating that the effect does not depend on anesthesia.

\section{Entrainment of slow oscillations}

Rhythmic stimulation-induced entrainment of neuronal oscillations has been observed in the cortex of human and nonhuman primates during behavioral tasks (Lakatos et al., 2005, 2008; Ding et al., 2006), a process thought to play a role in attentional modulation (Lakatos et al., 2008). Earlier studies using extracellular recordings have shown that sound stimulation can modulate the frequency of slow oscillations in nonlemniscal MGB neurons of anesthetized guinea pigs (He, 2003). Intracellular recordings from anesthetized animals in the present study further demonstrate that sound stimulation can entrain the up-to-down transi- tions of the membrane potential of these neurons. Moreover, our results indicate that the sound stimulus is effective in triggering a response only when these MGB neurons are in the up state. This differs from the bidirectional transitions of cerebellar Purkinje cells in response to air-puff stimulation of the vibrissae (Loewenstein et al., 2005) and the increased up-state durations of cortical cells induced by visual stimulation (Anderson et al., 2000). Note that in our extracellular recording experiments, sound stimuli increased the multiunit spiking activity (Fig. 4; supplemental Fig. 2 , available at www.jneurosci.org as supplemental material). Reconstruction of the tetrode track suggests that these multiunit responses were recorded from the lemniscal neurons. These observations suggest that although both the nonlemniscal and lemniscal neurons can be entrained by the sound stimuli, their entrained oscillations may have the opposite phase. Thus, the entrainment of slow oscillations by sensory inputs varies among different sensory circuits.

\section{Mechanism underlying entrainment}

Experimental studies (Sanchez-Vives and McCormick, 2000; Timofeev et al., 2000, 2001) and computational modeling (Bazhenov et al., 2002; Compte et al., 2003; Destexhe and Sejnowski, 


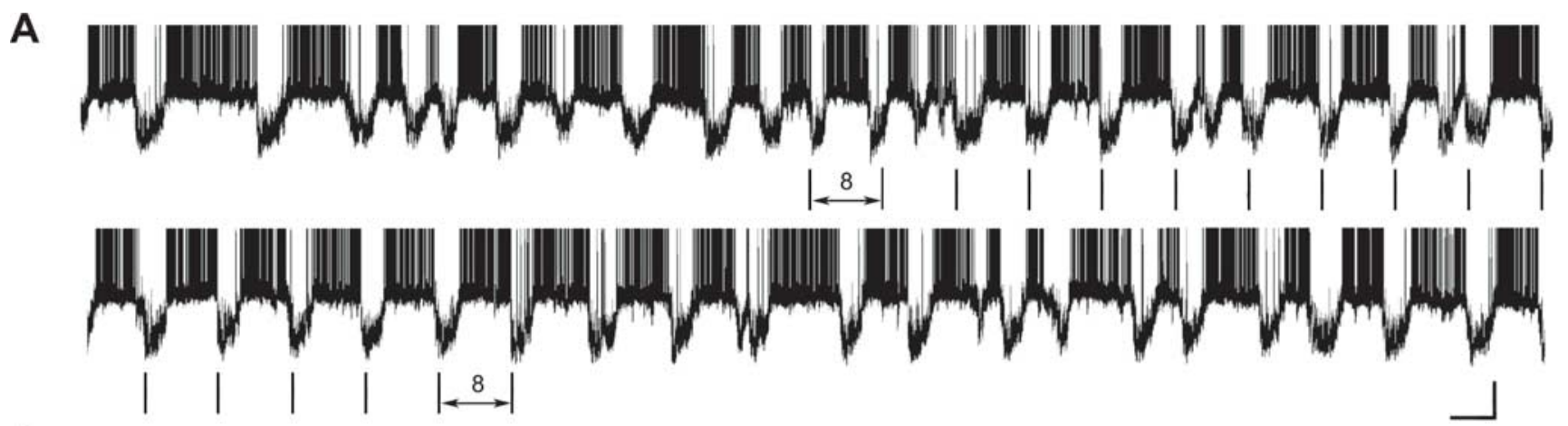

B

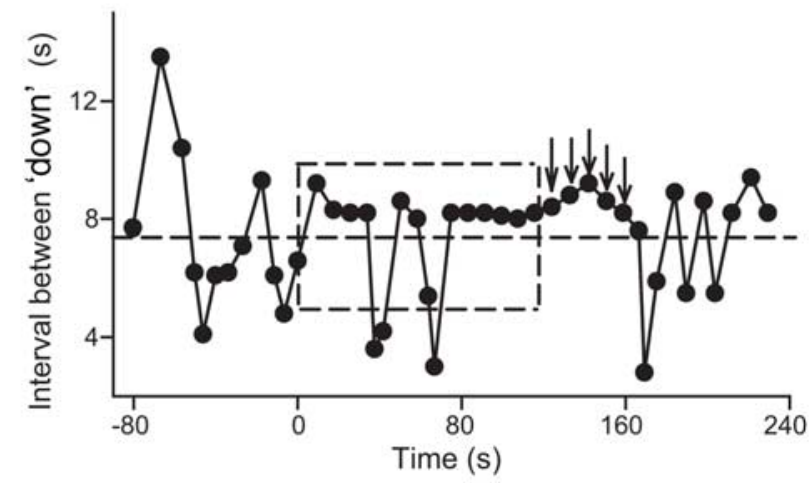

D1

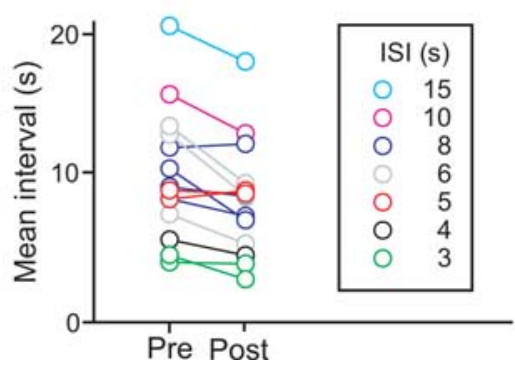

D2

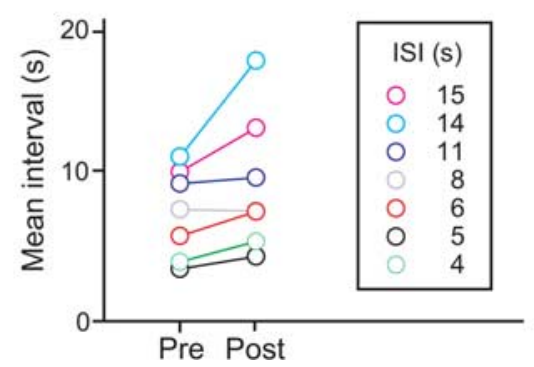

C

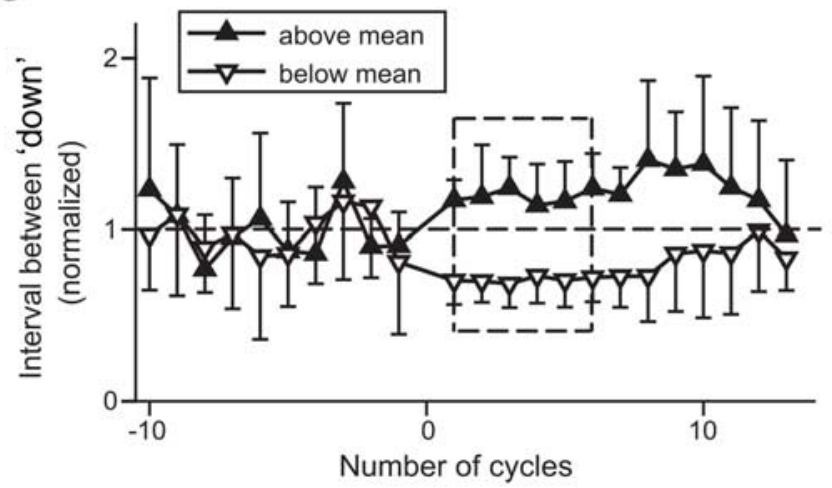

E

Figure 5. Short-term effect of periodic sound stimulation on spontaneous up/down transitions. $\boldsymbol{A}$, An example recording of membrane potentials before, during, and after periodic noise stimuli at 8 s interval. Calibration: $20 \mathrm{mV}, 5$ s. $\boldsymbol{B}$, Intervals between consecutive down states before, during, and after sound stimuli at an ISI longer than the average interval of spontaneous transitions before stimulation, for the cell shown in $\boldsymbol{A}$. Dashed line, mean interval between down states before stimulation. Rectangle marks the period of stimulation. Note that after the stimulation, the interval of spontaneous transitions remained longer than the average for several cycles (arrows). C, Summary of intervals between down states, for experiments with sound ISI longer (filled symbol) or shorter (open symbol) than the mean interval. Transition intervals for each cell were normalized by the mean before stimulation. Note that after stimulation, the mean transition interval was biased toward the sound ISIs for $\sim 7$ cycles. Error bar represents SD. D, Comparison between mean spontaneous transition intervals before (Pre) and after (Post) the periodic stimulation, for experiments with sound ISI shorter $\left(\boldsymbol{D}_{1}\right)$ or longer $\left(\boldsymbol{D}_{2}\right)$ than the mean spontaneous interval before (Pre) stimulation. Inset, ISI of the periodic stimuli indicated by number (in seconds) for each experiment, indicated by

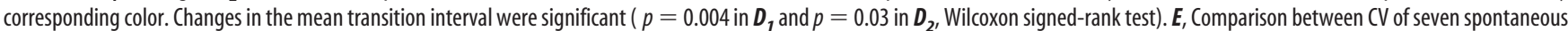
transition intervals before and after periodic sound stimulation. The reduction in CV after stimulation was significant ( $p=0.01$, Wilcoxon signed-rank test).

2003) have shown that thalamic oscillations depend on the interplay between intrinsic membrane conductances and synaptic activity. In the thalamocortical network, the slow oscillation involves a cortico-thalamo-cortical loop (Steriade et al., 1993a,c). The neocortex can generate slow oscillations spontaneously, depending on both the intrinsic neuronal excitability and intracortical inhibitory interactions (Steriade et al., 1993a; Sanchez-Vives and McCormick, 2000), whereas oscillations in the thalamus are triggered or terminated by corticothalamic feedback control, mainly through the activation of TRN (Huntsman et al., 1999; Bal et al., 2000; Steriade, 2001; Timofeev et al., 2001). Early studies using intracellular recordings have shown that the down state of neocortical neurons results from the absence of excitatory synaptic activity and was dominated by the $\mathrm{K}^{+}$currents (Steriade et al., 1993b; Timofeev et al., 2001). In contrast, our results suggest that the down state of nonlemniscal neurons is mainly mediated by $\mathrm{Cl}^{-}$currents (Fig. 3), presumably attributable to inhibitory inputs from the TRN or from the local interneurons in the MGB. The dependence of up/down transitions in neocortical and thalamic neurons on synaptic inputs is distinct from that found in cerebellar Purkinje cells, where membrane potential bistability is attributed entirely to intrinsic membrane properties (Williams et al., 2002; Loewenstein et al., 2005).

The mechanism underlying the entrainment of slow oscillations remains widely unknown. A recent study has shown that the aggregate locomotion behavior of a unicellular organism Physarum polycephalum can be entrained by periodic changes in environmental temperature and humidity with an ISI on the order of tens of minutes (Saigusa et al., 2008). The entrained behavior persists for 2-3 cycles after termination of the ES (a short-term 
effect), and a single pulse of stimulation could trigger several cycles of behavioral oscillation at the entrained interval several hours after the ES (a long-term effect). These short- and long-term effects are analogous to those shown in Figures 5 and 6 , respectively. Interestingly, both the short- and long-term effects can be explained by a simple dynamics model, in which periodic stimulation induces synchronization of multiple intrinsic oscillators within the aggregate. In neural circuits, both intrinsic neuronal properties (e.g., pace-maker activity) and synaptic interactions among neurons may give rise to entrainable oscillators. It would be of great interest to identify these entrainable oscillators in the thalamocortical loop and to elucidate the cellular mechanisms for retaining the information of the entrained ISI after conditioning.

\section{Functional implications}

The nonlemniscal thalamic neurons located in the nonprimary projection pathway are thought to be involved in temporal pattern recognition (Kraus et al., 1994; He et al., 1997), sound localization (Rauschecker, 1998), and some forms of learning-related plasticity (Steriade and Timofeev, 2003; Weinberger, 2004). Recent studies have also suggested that single nonlemniscal thalamic neurons can encode the timing of anticipated reward (Komura et al., 2001). Since slow oscillations of the membrane potential can regulate spiking responses to sensory inputs (Arieli et al., 1996; Azouz and Gray, 1999; Petersen et al., 2003; Sachdev et al., 2004), the entrainment effects reported here may provide a mechanism for modulating the processing of periodic sensory inputs, during both the entrainment process and the post-ES period. Indeed, we found that the efficacy of weak sound stimulus in triggering MGB responses was significantly enhanced at the entrained interval for at least 10 min after ES. Although in this study the entrainment effect was observed primarily under anesthesia and during slow wave sleep but not during passive wakefulness, it would be interesting for future studies to explore whether similar entrainment can be induced during certain behavioral tasks. Analogous entrainment effects by periodic sensory stimulation have recently been reported in zebrafish larvae (Sumbre et al., 2008), where conditioning visual stimulation at an interval of a few seconds induced rhythmic neuronal ensemble activity in the optic tectum that persisted for up to $20 \mathrm{~s}$ after termination of the visual stimulation. In this case, the entrained neuronal activity appears to have a direct behavioral consequence: it is correlated strongly with the zebrafish's visuomotor escape behavior after conditioning. min after ES.
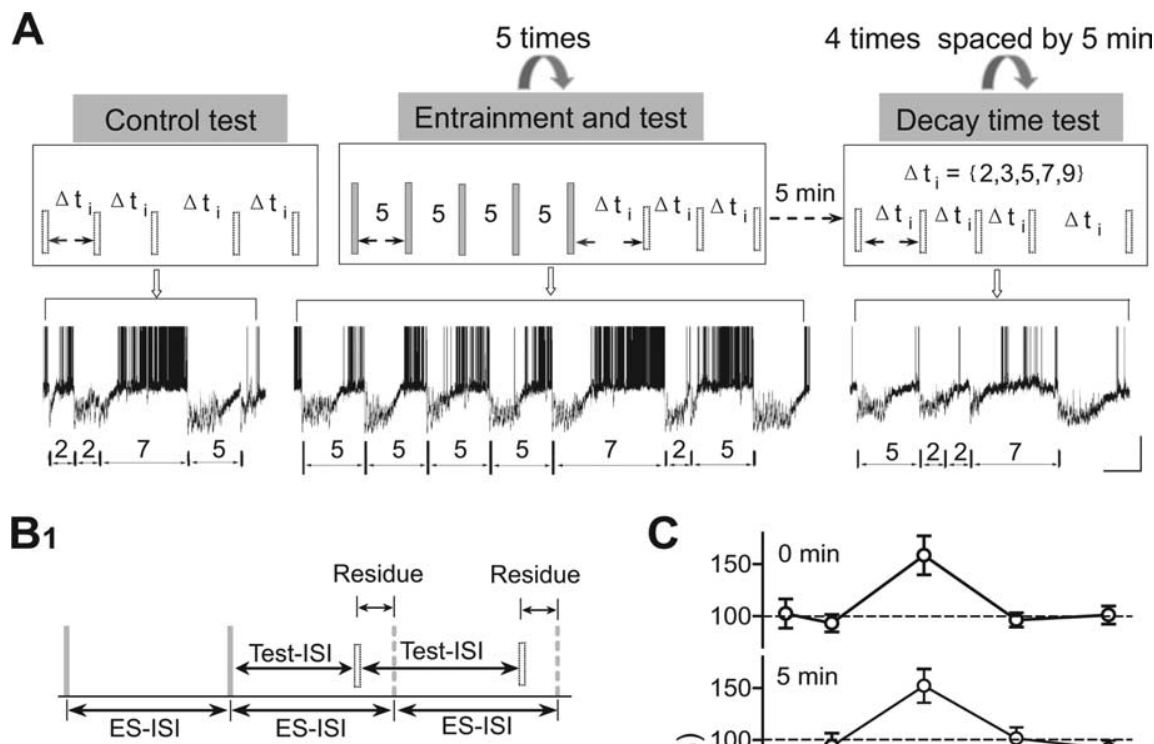

$\mathrm{B}_{2}$

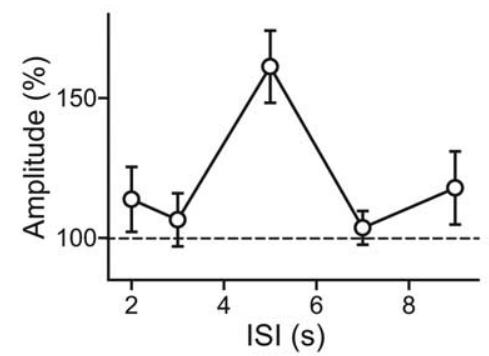

B3

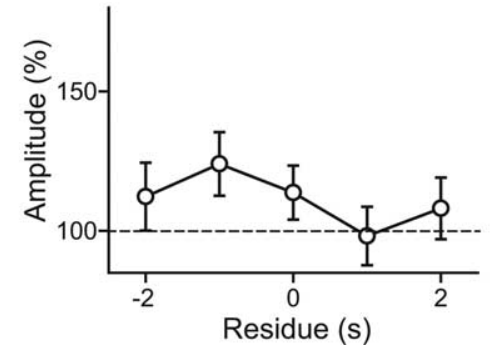

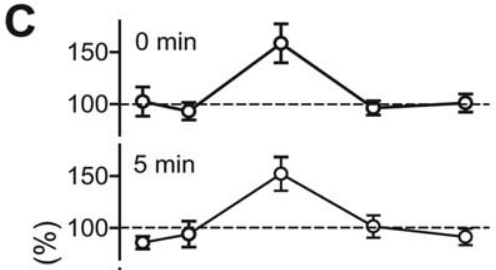
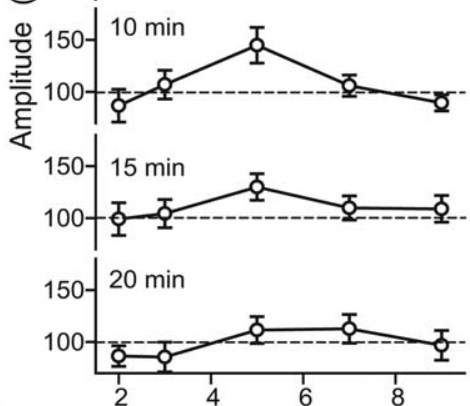

D

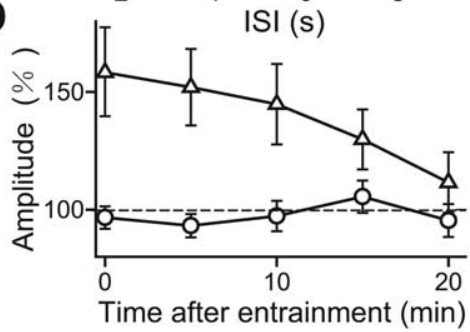

Figure 6. Effects of periodic sound stimulation on up-to-down transitions evoked by weak stimuli. $\boldsymbol{A}$, Schematic diagram of experimental procedure (top) and an example recording of responses to $\mathrm{ES}$ stimulation ( $5 \mathrm{~s} \mid \mathrm{SI}, 20 \mathrm{~dB}$ stronger than test stimuli) and test stimulation $(2,3,5,7$, and $9 \mathrm{~s}|S| s$; the temporal order of these ISIs was randomized in each trial). Calibration: $20 \mathrm{mV}, 3 \mathrm{~s}$. Long gray bar, ES. Short white bar, test stimulus. $\boldsymbol{B}$, Potentiation of the efficacy of the weak test stimulus at the entrained ISI. $\boldsymbol{B}_{\boldsymbol{1}}$, Definition of the test-ISI and the residue. Dashed gray bar, anticipated time of the ES after termination of the ES block. Test-ISI is defined as the interval between two consecutive test stimuli (short white bars). Residue is defined as the shortest interval between a given test stimulus and an anticipated time of the ES. $\boldsymbol{B}_{2}$, Amplitude of response evoked by the weak test stimulus (measured by area of down state over 1 s period, same as in Fig. $2 F$ ) immediately after ES as a function of test-ISI. For each cell, the response amplitude was normalized by that before ES at the same ISI. Error bars indicate SEM. The responses to test stimuli at the entrained ISI (5s) was significantly higher than $100 \%$ ( $p=0.0004$, paired $t$ test) and than the amplitudes at $3 \mathrm{~s}(p=0.005)$ and $7 \mathrm{~s}(p=$ 0.003). $\boldsymbol{B}_{3}$, Amplitude of response evoked by the weak test stimulus as a function of the residue. No significant difference was found between the amplitudes at -1 and $0 s$ ( $p=0.43$, paired $t$ test) or between 1 and $0 s(p=0.25)$. C, Same as $\boldsymbol{B}$, at different times after ES. $\boldsymbol{D}$, Normalized response amplitude as a function of time after ES for the entrained ISI (triangle) and the average of all other ISIs (circle). The increase in amplitude for entrained ISI was significant at $0(p=0.02), 5(p=0.02)$, and $10(p=0.02)$

In summary, our study showed that slow oscillations of neuronal activity are prevalent in the nonlemniscal thalamic nuclei, which can be entrained by periodic sensory stimuli. This may facilitate processing of rhythmic sensory inputs. In addition, we found that the entraining stimuli have significant effects on subsequent spontaneous slow oscillations and the efficacy of sensory stimuli in evoking responses. Such effects may help retain the information of the time interval of rhythmic sensory events. 


\section{References}

Anderson J, Lampl I, Reichova I, Carandini M, Ferster D (2000) Stimulus dependence of two-state fluctuations of membrane potential in cat visual cortex. Nat Neurosci 3:617-621.

Arieli A, Sterkin A, Grinvald A, Aertsen A (1996) Dynamics of ongoing activity: explanation of the large variability in evoked cortical responses. Science 273:1868-1871.

Azouz R, Gray CM (1999) Cellular mechanisms contributing to response variability of cortical neurons in vivo. J Neurosci 19:2209-2223.

Bal T, Debay D, Destexhe A (2000) Cortical feedback controls the frequency and synchrony of oscillations in the visual thalamus. J Neurosci 20:7478-7488.

Bazhenov M, Timofeev I, Steriade M, Sejnowski TJ (2002) Model of thalamocortical slow-wave sleep oscillations and transitions to activated States. J Neurosci 22:8691-8704.

Compte A, Sanchez-Vives MV, McCormick DA, Wang XJ (2003) Cellular and network mechanisms of slow oscillatory activity $(<1 \mathrm{~Hz})$ and wave propagations in a cortical network model. J Neurophysiol 89:2707-2725.

Destexhe A, Sejnowski TJ (2003) Interactions between membrane conductances underlying thalamocortical slow-wave oscillations. Physiol Rev 83:1401-1453.

Ding J, Sperling G, Srinivasan R (2006) Attentional modulation of SSVEP power depends on the network tagged by the flicker frequency. Cereb Cortex 16:1016-1029.

He J (2003) Slow oscillation in non-lemniscal auditory thalamus. J Neurosci 23:8281-8290.

He J, Hashikawa T, Ojima H, Kinouchi Y (1997) Temporal integration and duration tuning in the dorsal zone of cat auditory cortex. J Neurosci 17:2615-2625.

Huntsman MM, Porcello DM, Homanics GE, DeLorey TM, Huguenard JR (1999) Reciprocal inhibitory connections and network synchrony in the mammalian thalamus. Science 283:541-543.

Kitano K, Câteau H, Kaneda K, Nambu A, Takada M, Fukai T (2002) Twostate membrane potential transitions of striatal spiny neurons as evidenced by numerical simulations and electrophysiological recordings in awake monkeys. J Neurosci 22:RC230.

Komura Y, Tamura R, Uwano T, Nishijo H, Kaga K, Ono T (2001) Retrospective and prospective coding for predicted reward in the sensory thalamus. Nature 412:546-549.

Kraus N, McGee T, Littman T, Nicol T, King C (1994) Nonprimary auditory thalamic representation of acoustic change. J Neurophysiol 72:1270-1277.

Lakatos P, Shah AS, Knuth KH, Ulbert I, Karmos G, Schroeder CE (2005) An oscillatory hierarchy controlling neuronal excitability and stimulus processing in the auditory cortex. J Neurophysiol 94:1904-1911.

Lakatos P, Karmos G, Mehta AD, Ulbert I, Schroeder CE (2008) Entrainment of neuronal oscillations as a mechanism of attentional selection. Science 320:110-113.

Loewenstein Y, Mahon S, Chadderton P, Kitamura K, Sompolinsky H, Yarom
Y, Häusser M (2005) Bistability of cerebellar Purkinje cells modulated by sensory stimulation. Nat Neurosci 8:202-211.

Petersen CC, Hahn TT, Mehta M, Grinvald A, Sakmann B (2003) Interaction of sensory responses with spontaneous depolarization in layer $2 / 3$ barrel cortex. Proc Natl Acad Sci U S A 100:13638-13643.

Rauschecker JP (1998) Parallel processing in the auditory cortex of primates. Audiol Neurootol 3:86-103.

Sachdev RN, Ebner FF, Wilson CJ (2004) Effect of subthreshold up and down states on the whisker-evoked response in somatosensory cortex. J Neurophysiol 92:3511-3521.

Saigusa T, Tero A, Nakagaki T, Kuramoto Y (2008) Amoebae anticipate periodic events. Phys Rev Lett 100:018101.

Sanchez-Vives MV, McCormick DA (2000) Cellular and network mechanisms of rhythmic recurrent activity in neocortex. Nat Neurosci 3:1027-1034.

Schonewille M, Khosrovani S, Winkelman BH, Hoebeek FE, De Jeu MT, Larsen IM, Van der Burg J, Schmolesky MT, Frens MA, De Zeeuw CI (2006) Purkinje cells in awake behaving animals operate at the upstate membrane potential. Nat Neurosci 9:459-461; author reply 461.

Steriade M (2001) The GABAergic reticular nucleus: a preferential target of corticothalamic projections. Proc Natl Acad Sci U S A 98:3625-3627.

Steriade M, Timofeev I (2003) Neuronal plasticity in thalamocortical networks during sleep and waking oscillations. Neuron 37:563-576.

Steriade M, McCormick DA, Sejnowski TJ (1993a) Thalamocortical oscillations in the sleeping and aroused brain. Science 262:679-685.

Steriade M, Nuñez A, Amzica F (1993b) A novel slow ( $<1 \mathrm{~Hz}$ ) oscillation of neocortical neurons in vivo: depolarizing and hyperpolarizing components. J Neurosci 13:3252-3265.

Steriade M, Contreras D, Curró Dossi R, Nuñez A (1993c) The slow $(<1$ $\mathrm{Hz}$ ) oscillation in reticular thalamic and thalamocortical neurons: scenario of sleep rhythm generation in interacting thalamic and neocortical networks. J Neurosci 13:3284-3299.

Sumbre G, Muto A, Baier H, Poo MM (2008) Entrained rhythmic activities of neuronal ensembles as perceptual memory of time interval. Nature 456:102-106.

Timofeev I, Grenier F, Bazhenov M, Sejnowski TJ, Steriade M (2000) Origin of slow cortical oscillations in deafferented cortical slabs. Cereb Cortex 10:1185-1199.

Timofeev I, Grenier F, Steriade M (2001) Disfacilitation and active inhibition in the neocortex during the natural sleep-wake cycle: an intracellular study. Proc Natl Acad Sci U S A 98:1924-1929.

Weinberger NM (2004) Specific long-term memory traces in primary auditory cortex. Nat Rev Neurosci 5:279-290.

Williams SR, Christensen SR, Stuart GJ, Häusser M (2002) Membrane potential bistability is controlled by the hyperpolarization-activated current $\mathrm{I}(\mathrm{H})$ in rat cerebellar Purkinje neurons in vitro. J Physiol 539:469-483.

Wilson CJ, Kawaguchi Y (1996) The origins of two-state spontaneous membrane potential fluctuations of neostriatal spiny neurons. J Neurosci 16: 2397-2410. 